\title{
Fundamental Trade-Offs in Aggregate Packet Scheduling
}

\author{
Zhenhai Duan, Member, IEEE, Zhi-Li Zhang, Member, IEEE, and \\ Yiwei Thomas Hou, Senior Member, IEEE
}

\begin{abstract}
In this paper, we investigate the fundamental trade-offs in aggregate packet scheduling for support of guaranteed delay service. In our study, we consider two classes of aggregate packet scheduling algorithms: the static earliest time first (SETF) and dynamic earliest time first (DETF). Through these two classes of aggregate packet scheduling (and together with the simple FIFO packet scheduling algorithm), we show that, with additional timestamp information encoded in the packet header for scheduling purposes, we can significantly increase the maximum allowable network utilization level, while, at the same time, reducing the worstcase edge-to-edge delay bound. Furthermore, we demonstrate how the number of the bits used to encode the timestamp information affects the trade-off between the maximum allowable network utilization level and the worst-case edge-to-edge delay bound. In addition, the more complex DETF algorithms have far superior performance than the simpler SETF algorithms. These results illustrate the fundamental trade-offs in aggregate packet scheduling algorithms and shed light on their provisioning power in support of guaranteed delay service.
\end{abstract}

Index Terms-Packet scheduling algorithms, aggregate packet scheduling algorithms, quality of services (QoS), performance analysis.

\section{INTRODUCTION}

$\mathrm{B}_{\mathrm{C}}^{\mathrm{E}}$ ECAUSE of its potential scalability in support of Internet QoS guarantees, lately, aggregate packet scheduling has attracted a lot of attention in the networking community. For instance, in the DiffServ framework [2], it is proposed that the simple FIFO packet scheduling be used to support the EF (expedited forwarding) per-hop behavior (PHB) [8]. Namely, at each router, EF packets from all users are queued at a single FIFO buffer and serviced in the order of their arrival times at the queue. Clearly, use of FIFO packet scheduling results in a very simple implementation of the EF PHB. However, the ability to appropriately provision a network using FIFO packet scheduling to provide guaranteed rate/delay service-as the EF PHB is arguably intended to support [9] — has been questioned [1], [6].

In a recent work by Charny and Le Boudec [6], it is shown that, in order to provide guaranteed delay service using FIFO, the overall network utilization level should be limited to a small fraction of its link capacities. More specifically, in a network of FIFO schedulers, a bound on the worst-case delay at each router is derived only when the network utilization level is limited to a factor smaller than $1 /\left(H^{*}-1\right)$, where $H^{*}$, referred to as the network diameter, is the number of hops in the longest path of the network. Furthermore, given the network

- Z. Duan is with the Computer Science Department, Florida State University, Tallahassee, FL 32306. E-mail: duan@cs.fsu.edu.

- Z.-L. Zhang is with the Department of Computer Science and Engineering, University of Minnesota, Minneapolis, MN 55455.

E-mail: zhzhang@cs.umn.edu.

- Y.T. Hou is with the Bradley Department of Electrical and Computer Engineering, Virginia Tech, Blacksburg, VA 24061.

E-mail: thou@vt.edu.

Manuscript received 3 Sept. 2003; revised 16 Jan. 2005; accepted 23 Mar. 2005; published online 20 Oct. 2005.

For information on obtaining reprints of this article, please send e-mail to: tpds@computer.org, and reference IEEECS Log Number TPDS-0152-0903. utilization level $\alpha<1 /\left(H^{*}-1\right)$, the derived worst-case delay bound is inversely proportional to $1-\alpha\left(H^{*}-1\right)$. Hence, as the network utilization level $\alpha$ gets closer to the utilization bound $1 /\left(H^{*}-1\right)$, the worst-case delay bound rapidly approaches infinity.

The elegant result of Charny and Le Boudec raises several interesting and important questions regarding the design and provisioning power of aggregate packet scheduling. In this paper, we will take a more theoretical perspective and attempt to address the fundamental tradeoffs in the design of aggregate packet scheduling algorithms and their provisioning power in support of (worst-case) guaranteed delay service. In particular, we study the relationships between the worst-case edge-to-edge delay (i.e., the maximum delay experienced by any packet across a network domain), the maximum allowable network utilization level, and the "sophistication/complexity" of aggregate packet scheduling employed by a network. À la the Internet DiffServ paradigm, we consider a framework where user traffic is only conditioned (i.e., shaped) at the edge of a network domain, whereas, inside the network core, packets are scheduled based solely on certain bits (referred to as the packet state carried in the packet header). In other words, the aggregate packet scheduling algorithm employed inside the network core maintains no per-flow/ user information, thus it is core-stateless.

In our framework, besides the conventional "Type of Service (TOS)" or "Differentiated Service (DS)" bits, we assume that additional control information may be carried in the packet header for scheduling purpose. By encoding certain timing information in the packet header, we design two classes of aggregate packet scheduling algorithms: the static earliest time first (SETF) and dynamic earliest time first (DETF) algorithms. In the class of SETF packet scheduling 
TABLE 1

Notations Used in This Paper

\begin{tabular}{|c|c|}
\hline$H^{*}$ & Network diameter \\
\hline$\alpha$ & Network utilization level \\
\hline$\beta$ & Traffic burstiness \\
\hline$\left(r_{S}, e_{S}\right)$ & Rate and latency parameters of a GR server $S$ \\
\hline$e$ & $e=\max _{\text {all } S^{\prime}{ }_{s}}\left\{e_{S}\right\}$ \\
\hline$\Delta$ & maximum transmission time of an arbitrary packet \\
\hline$\Gamma$ & Duration of a time slot \\
\hline$h^{*}$ & $\begin{array}{l}\text { Maximum number of hops that a packet can traverse within } \Gamma \text { (in } \operatorname{SETF}(\Gamma) \text { ); } \\
\text { Hop count when a packet's time stamp needs to be updated (in DETF) }\end{array}$ \\
\hline$D_{H^{*}}$ & Worst-case end-to-end delay bound \\
\hline$m$ & Number of bits needed to encode time stamp information \\
\hline$C^{*}$ & Maximum link capacity in a network \\
\hline$\iota$ & $\iota=1 / C^{*}$, finest time granularity \\
\hline$d^{*}$ & Time stamp increment in DETF \\
\hline
\end{tabular}

algorithms, packets are stamped with their entry time at the network edge and they are scheduled in the order of their timestamps (i.e., their network entry times) inside the network core; the class of DETF packet scheduling algorithms work in a similar fashion, albeit with an important difference-the packet timestamps are updated at certain routers (hence, the term dynamic). In both classes, the granularity of timing information encoded in the packet state-as is determined by the number of bits used for packet state encoding-is a critical factor that affects the provisioning power of aggregate packet scheduling.

The contribution of our study is that, using these two classes (SETF and DETF) of aggregate packet scheduling algorithms, in addition to the simple FIFO discipline, we explore the fundamental trade-offs in aggregate packet scheduling:

- how, with additional control information encoded in the packet state and with added "sophistication/ complexity" in aggregate packet scheduling, the worst-case edge-to-edge delay bound and the maximum allowable network utilization bound can be improved, and

- how these performance bounds are affected by the number of bits available for packet state encoding.

Through analysis and numerical examples, we show that, when packet timestamps are encoded with the finest time

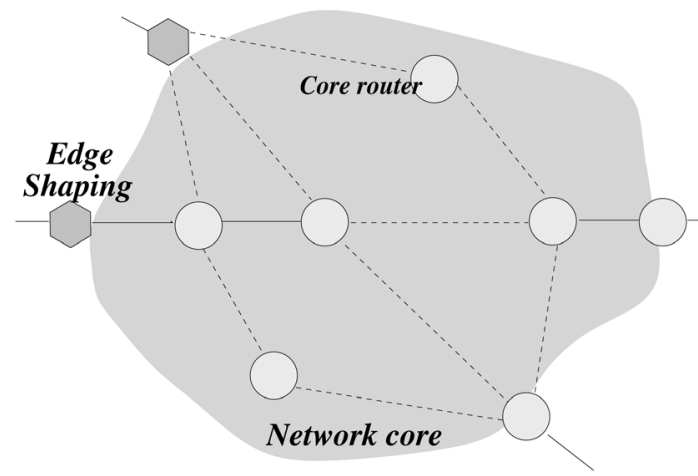

Fig. 1. The network model. granularity, i.e., carrying the precise releasing time of the packets, both the SETF and DETF packet scheduling algorithms can attain an arbitrary network utilization level (i.e., $\alpha$ can be arbitrarily close to 1). In other words, the maximum allowable network utilization bound is independent of the network diameter $H^{*}$. This is in contrast to the case of FIFO, where the known maximum utilization level is bounded by $1 /\left(H^{*}-1\right)$. Furthermore, using the more complex DETF, the worst-case edge-to-edge delay bound is linear in $H^{*}$, whereas, using the simpler SETF, the worst-case edge-toedge delay bound is inversely proportional to $(1-\alpha)^{H^{*}}$. When packet timestamps are encoded using coarser granularity (i.e., the number of bits for packet state encoding is limited), the network utilization level is constrained by the time granularity. In addition, the worst-case edge-to-edge delay bound is increased. With the same number of bits, the more complex DETF packet scheduling algorithms have far superior performance over the simpler SETF algorithms.

The remainder of the paper is organized as follows: In Section 2, we present the basic model and assumptions for our analysis. The two classes of aggregate packet scheduling, SETF and DETF, are analyzed and the trade-offs discussed in Section 3 and Section 4, respectively. We conclude the paper in Section 5.

\section{Network Model and Assumptions}

Before we start, it is worth noting that all the major notations used in this paper are summarized in Table 1.

Consider a single network domain, as shown in Fig. 1, where we distinguish network edge routers from core routers. We assume that a number of traffic classes (or aggregates) are supported by the network domain. Conceptually, we can imagine that there is a separate queue for each traffic class at a core router. We further assume that, for each traffic aggregate, the router provides a Guaranteed Rate (GR) service curve (also called rate-latency service curve) [4] to the aggregate. In particular, if a router provides a rate-latency service curve $\delta(t)=r_{d}\left(t-e_{d}\right)^{+}$to a traffic aggregate, we would say that, for the traffic aggregate, the router is a GR node with parameters $\left(r_{d}, e_{d}\right)$. We refer to $r_{d}$ and $e_{d}$ as the reserved rate and latency for the aggregate at 


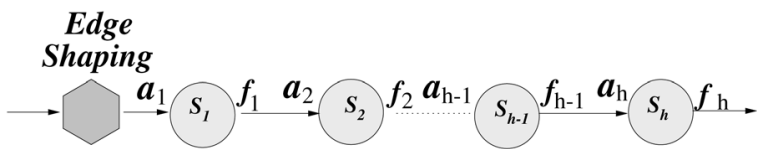

Fig. 2. Packet's arrival time at and departure time from each scheduler.

the router, respectively. Note that this definition of the GR node is independent of the kind of scheduling discipline used for scheduling packets within the traffic aggregate. We call this scheduling discipline for packets within the aggregate packet scheduling discipline and assume that, for a traffic aggregate, all routers employ the same aggregate packet scheduling algorithm (e.g., FIFO) that performs packet scheduling using only certain bits (the packet state) carried in the packet header. No other scheduling information is used or stored at core routers. We refer to the aggregate scheduling mechanism employed at an outgoing link of a router ${ }^{1}$ as a scheduler. For clarity, we may incorporate the name of aggregate packet scheduling discipline into the notion of the GR node. For example, if the FIFO discipline is used for a traffic aggregate and the aggregate receives a GR service curve with parameters $\left(r_{d}, e_{d}\right)$ at a router, then we would refer to this router as a GR-FIFO node (scheduler) with parameters $\left(r_{d}, e_{d}\right)$ (or simply FIFO node with parameters $\left(r_{d}, e_{d}\right)$ when there is no confusion) for the traffic aggregate. In this paper, we only focus on the aggregate packet scheduling discipline for a single traffic aggregate. Without loss of generality, the remaining discussions in the paper are all related to this single traffic aggregate (e.g., an EF aggregate). Moreover, we assume that all traffic of the aggregate entering the network is shaped at the edge traffic conditioner before releasing into the network. No traffic shaping or reshaping is performed inside the network core.

Consider a GR scheduler $S$ with parameters $\left(r_{S}, e_{S}\right)$. We denote the MTU (maximum transmission unit) of the link by $L_{S}^{\max }$, then $\Delta_{S}=L_{S}^{\max } / r_{S}$ is the transmission time of an MTU-sized packet with the reserved rate $r_{S}$. Define $\Delta=$ $\max _{\text {all } S^{\prime} s}\left\{\Delta_{S}\right\}$ and $e=\max _{\text {all } S^{\prime} s}\left\{e_{S}\right\}$. We also assume that the path of any user flow is predetermined and fixed throughout its duration. Let $H^{*}$ be the maximum number of hops in the paths that any user flow may traverse in the network. We refer to $H^{*}$ as the network diameter.

Consider an arbitrary flow $j$ (of the aggregate) traversing the network. The traffic of the flow is shaped at the network edge in such a manner that it conforms to a token bucket regulated arrival curve $\left(\sigma^{j}, \rho^{j}\right)$ [7]: Let $A^{j}(t, t+\tau)$ denote the amount of the flow $j$ traffic released into the network during a time interval $[t, t+\tau]$, where $t \geq 0, \tau \geq 0$; then $A^{j}(t, t+\tau)$ $\leq \sigma^{j}+\rho^{j} \tau$. We control the overall network utilization level by imposing a utilization factor $\alpha$ on each link as follows (with respect to the reserved rate of the aggregate): Consider an arbitrary GR scheduler $S$ with parameters $\left(r_{S}, e_{S}\right)$. Let $\mathcal{F}$ denote the set of user flows traversing $S$ in the aggregate. Then, the following condition holds:

$$
\sum_{j \in \mathcal{F}} \rho^{j} \leq \alpha r_{S}
$$

1. For simplicity, we assume that output-queuing is used. where $0<\alpha \leq 1$. We will also refer to the utilization factor $\alpha$ as the network utilization level of a network domain. In addition to the link utilization factor $\alpha$, we will also impose an overall bound $\beta \geq 0$ (in units of time) on the "burstiness" of flows traversing scheduler $S: \sum_{j \in \mathcal{F}} \sigma^{j} \leq \beta r_{S}$. As we will see later, this burstiness factor $\beta$ plays a less critical role in our analysis than the network utilization level $\alpha$. It is worth noting that both the network utilization level $\alpha$ and the burstiness factor $\beta$ are defined with respect to the reserved rate of an aggregate instead of link capacities.

From the above edge shaping and network utilization constraints, we can obtain an important bound on the amount of traffic of the aggregate going through a given scheduler that is injected at the network edge during any time interval. Consider an arbitrary GR scheduler $S$ with parameters $\left(r_{S}, e_{S}\right)$. For any time interval $[\tau, t]$, let $\AA_{S}(\tau, t)$ denote the amount of traffic of the aggregate injected into the network during the time interval $[\tau, t]$ that will traverse $S$ (at perhaps some later time). Here, we use $\AA$ to emphasize that $\AA_{S}(\tau, t)$ is not the traffic traversing $S$ during the time interval $[\tau, t]$, but injected into the network at the network edge during $[\tau, t]$. Using the facts that $A^{j}(t, t+\tau) \leq \sigma^{j}+\rho^{j} \tau$ for all flows, $\sum_{j \in \mathcal{F}} \rho^{j} \leq \alpha r_{S}$ and $\sum_{j \in \mathcal{F}} \sigma^{j} \leq \beta r_{S}$, it is easy to show that

$$
\AA_{S}(\tau, t) \leq \alpha r_{S}(t-\tau)+\beta r_{S} .
$$

We refer to this bound as the edge traffic provisioning condition for scheduler $S$. As we will see later, the edge traffic provisioning condition is critical to our analysis of aggregate packet scheduling algorithms.

Now, consider a packet $p$ (of any flow) that traverses a path with $h^{p} \leq H^{*}$ hops. For $i=1,2, \ldots, h^{p}$, denote the scheduler at the $i$ th hop on the path of packet $p$ as $S_{i}$ (see Fig. 2). Let $a_{i}^{p}$ and $f_{i}^{p}$ represent, respectively, the time that packet $p$ arrives at and departs ${ }^{2}$ from scheduler $S_{i}$. For ease of exposition, throughout this paper, we assume that the propagation delay from one scheduler to another scheduler is zero. Hence, $a_{i+1}^{p}=f_{i}^{p}$. Note that $a_{1}^{p}$ is the time packet $p$ is released into the network (after going through the edge traffic conditioner). Define $d_{i}^{p}=f_{i}^{p}-a_{1}^{p}$, i.e., $d_{i}^{p}$ is the delay experienced by the packet after traversing the $i$ th hop. (Note that the delay experienced by a packet at the edge traffic conditioner is excluded from the edge-to-edge delay.) Hence, $d_{h^{p}}^{p}$ is the cumulative delay that packet $p$ experiences along its path and is referred to as the edge-to-edge delay experienced by packet $p$. Define $D_{i}$ to be the worst-case edge-to-edge delay experienced by any packet in the network after traversing $i$ hops,

$$
D_{i}=\max _{\text {all } p^{\prime} s \text { with } h^{p} \geq i}\left\{d_{i}^{p}\right\} .
$$

Therefore, $D_{H^{*}}$ is the worst-case edge-to-edge delay experienced by any packet in the aggregate in the network.

The key questions that we will address in the remainder of the paper are: 1) Given an aggregate packet scheduling algorithm, under what network utilization level $\alpha$ does an upper bound on $D_{H^{*}}$ exist? 2) How does this bound depend

2. Throughout the paper, we adopt the following convention: A packet is considered to have arrived at a scheduler only when its last bit has been received and it is considered to have departed from the scheduler only when its last bit has been serviced. 
on the network utilization level $\alpha$ and the network diameter $H^{*}$ ? 3) How are these relationships affected by the number of bits available for packet state encoding as well as the added "sophistication/complexity" in aggregate packet scheduling?

In order to compare with the new aggregate packet scheduling disciplines that we will study in this paper and to illustrate the trade-offs in designing aggregate scheduling, we restate the performance bounds of a general FIFO network by Charny and Le Boudec here [6], using the above set of notation, before we leave this section.

Theorem 1. Given a network of FIFO schedulers with a network diameter $H^{*}$, if the network utilization level $\alpha$ satisfies the condition $\alpha<\frac{1}{H^{*}-1}$, then the worst-case edge-to-edge delay $D_{H^{*}}$ is bounded above by

$$
D_{H^{*}} \leq \frac{H^{*}(e+\beta)}{1-\left(H^{*}-1\right) \alpha}
$$

\section{Network of Static Earliest Time First SCHEDULERS}

In this section, we will design and analyze a new class of aggregate packet scheduling algorithms-the class of static earliest time first (SETF) algorithms. Using this class of aggregate packet scheduling algorithms, we will demonstrate how, by adding some "sophistication/complexity" in aggregate packet scheduling -in particular, by encoding additional control information in the packet header, we can improve the maximum allowable utilization bound and reduce the provable worst-case edge-to-edge delay bound, compared with the FIFO scheduling discipline. Furthermore, we will discuss the performance trade-offs of SETF packet algorithms when a limited number of bits is used for packet state encoding.

The additional control information used by the class of SETF schedulers is a (static) timestamp carried in the packet header of a packet that records the time the packet is released into the network (after going through the edge traffic conditioner) at the network edge. Here, we assume that all edge devices that timestamp the packets use a global clock (in other words, the clocks at the edge devices are synchronized). We denote the timestamp of a packet $p$ by $\omega_{0}^{p}$. An SETF scheduler inside the network core schedules packets in the order of their timestamps, $\omega_{0}^{p}$. Note that, in the case of SETF, the timestamp of a packet is never modified by any SETF scheduler, thus the term static.

Depending on the time granularity used to represent the packet timestamps, we can design a class of SETF schedulers with different performance/complexity tradeoffs. We use $\operatorname{SETF}(\Gamma)$ to denote the SETF packet scheduling algorithm where packet timestamps are represented with time granularity $\Gamma$. In particular, $\operatorname{SETF}(0)$ denotes the SETF packet scheduling algorithm where packet timestamps are represented with the finest time granularity, namely, packets are timestamped with the precise time they are released into the network. Formally, for any packet $p$, we have $\omega_{0}^{p}=a_{1}^{p}$. For a more general $\operatorname{SETF}(\Gamma)$ scheduling algorithm where $\Gamma>0$, we divide the time into slots of $\Gamma$

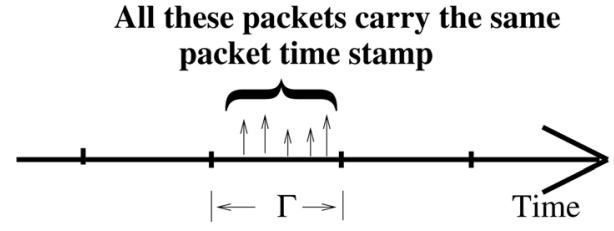

Fig. 3. Time slots and packet timestamps.

time units each (see Fig. 3): $t_{n}=[(n-1) \Gamma, n \Gamma), n=1,2, \ldots$ Packets released into the network are timestamped with the corresponding time slot number $n$. In other words, packets that are released into the network within the same time slot (say, the time slot $t_{n}=[(n-1) \Gamma, n \Gamma)$ ) carry the same timestamp value, i.e., $\omega_{0}^{p}=n$. Therefore, packets released into the network during the same time slot at the network edge are indistinguishable by an $\operatorname{SETF}(\Gamma)$ scheduler inside the network core, and are serviced by the scheduler in a FIFO manner. We will show later that using coarser time granularity (i.e., larger $\Gamma$ ) can potentially reduce the number of bits needed to encode the packet timestamps, but at the expenses of degrading the performance bounds.

In the rest of this section, we first establish the performance bounds for SETF and then discuss the packet state encoding issue. At the end of this section, we conduct numerical studies to illustrate the performance trade-offs and provisioning power of SETF.

\subsection{Performance Bounds for a Network of SETF Schedulers}

Note that $\operatorname{SETF}(0)$ can be considered as a special case of $\operatorname{SETF}(\Gamma)$, where $\Gamma=0$. Therefore, we focus on deriving the performance bounds for a network of $\operatorname{SETF}(\Gamma)$. The bounds for $\operatorname{SETF}(0)$ are presented thereafter as a corollary. Consider a network of $\operatorname{SETF}(\Gamma)$ schedulers. Recall that, under $\operatorname{SETF}(\Gamma)$, the time is divided into time slots and packets released into the network during the same time slot carry the same timestamp value (i.e., the time slot number). Clearly, the coarser the time granularity $\Gamma$ is, the more packets will be timestamped with the same time slot number. In particular, if $\Gamma$ is larger than the worst-case edge-to-edge delay of the network, then a network of $\operatorname{SETF}(\Gamma)$ schedulers degenerates to a network of FIFO schedulers.

Before we present the performance bounds for a network of $\operatorname{SETF}(\Gamma)$ schedulers, we first introduce a new notation, $h^{*}$ : For a given $\Gamma$, define $h^{*}+1$ to be the maximum number of hops that any packet can reach within $\Gamma$ units of time after it is released into the network. Mathematically, $h^{*}$ is the smallest $h$ such that the following relation holds for all packets:

$$
\min _{\text {all }}\left\{a_{h^{\prime} s}^{p}-a_{1}^{p}\right\} \geq \Gamma .
$$

The definition $h^{*}$ also presents an intuitive guideline for the amount of traffic that may interfere with each other. Consider an arbitrary packet $p$ and arbitrary GR-SETF $(\Gamma)$ scheduler $S$ along its path, then we know that a packet entering the network in the time interval $\left[a_{h^{*}+1}^{p}, a_{S}^{p}\right]$ must have a timestamp that is larger than that of packet $p$. Therefore, largely speaking, these packet will not interfere with the 
scheduling of packet $p$ at scheduler $S$. On the other hand, for $i \leq h^{*}$, packets entering the network after $a_{1}^{p}$ but before $a_{i}^{p}$ may have the same timestamp as packet $p$ and compete with packet $p$ for service. Note that if $h^{*}=0$, we must have $\Gamma=0$. This gives us $\operatorname{SETF}(0)$. On the other hand, if $\Gamma$ is large enough such that $h^{*}=H^{*}-1, \operatorname{SETF}(\Gamma)$ becomes FIFO.

We state the performance bounds for a network of GR$\operatorname{SETF}(\Gamma)$ schedulers in the following theorem [13].

Theorem 2. Consider a network of GR-SETF( $\Gamma)$ schedulers with a network diameter $H^{*}$. If the network utilization level $\alpha$ satisfies the following condition,

$$
(1-\alpha)^{H^{*}-h^{*}-1}>\alpha h^{*},
$$

then the worst-case edge-to-edge delay is bounded above by,

$$
D_{H^{*}} \leq \frac{(\beta+e) h^{*}+\alpha^{-1}(\beta+e+\Delta)\left\{1-(1-\alpha)^{H^{*}-h^{*}}\right\}}{(1-\alpha)^{H^{*}-h^{*}-1}-\alpha h^{*}} .
$$

By setting $h^{*}=0$ in Theorem 2, we obtain the performance bounds for a network of $\operatorname{SETF}(0)$ schedulers (see Theorem 3 below), whereas, letting $h^{*}=H^{*}-1$, we have the results for a network of FIFO schedulers (with a difference of $\frac{\Delta}{1-\left(H^{*}-1\right) \alpha}$ caused by the extra care taken by the analysis of an SETF network to account for the nonpreemptive property of an SETF scheduler, see Theorem 1 in Section 2).

Theorem 3. Consider a network of GR-SETF(0) schedulers with a network diameter $H^{*}$. The worst-case edge-to-edge delay, $D_{H^{*}}$, is bounded above by

$$
D_{H^{*}} \leq \frac{\alpha^{-1}(\beta+e+\Delta)\left\{1-(1-\alpha)^{H^{*}}\right\}}{(1-\alpha)^{H^{*}-1}}, 0<\alpha<1 .
$$

Comparing with a network of FIFO schedulers, we see that, in a network of $\operatorname{SETF}(0)$ schedulers, the network utilization level can be kept as high (i.e., as close to 1) as wanted: Unlike FIFO, there is no limit on the maximum allowable network utilization level. However, since the worst-case edge-to-edge delay bound is inversely proportional to $(1-\alpha)^{H^{*}-1}$, it increases exponentially as $\alpha \rightarrow 1$. On the other hand, in general, Theorem 2 states that, with a coarser time granularity $\Gamma>0$ (which determines $h^{*}$ ), we may no longer be able to set the network utilization level at any arbitrary level, as in the case of $\operatorname{SETF}(0)$, while still having a finite worst-case edge-to-edge delay bound. In other words, for a given $\Gamma>0$, there is a limit on the maximum allowable network utilization level as imposed by (6). This limit on the maximum allowable network utilization level is the performance penalty we pay for using coarser time granularity to represent the packet timestamp information. We will conduct numerical studies in Section 3.3 to illustrate these performance trade-offs.

\subsection{Time Stamp Encoding}

In this section, we discuss the implication of the worst-case edge-to-edge delay bound on the number of bits needed to encode the timestamp information. Again, we consider a network of $\operatorname{SETF}(\Gamma)$ first. Suppose that $m$ bits are sufficient to encode the packet timestamps precisely. Then, the timestamp bit string wraps around every $2^{m} \Gamma$ units of time.
Given that the worst-case edge-to-edge delay of a packet in the network of $\operatorname{SETF}(\Gamma)$ is bounded above by $D_{H^{*}}$, we must have $2 D_{H^{*}} \leq 2^{m} \Gamma$ so as to enable any $\operatorname{SETF}(\Gamma)$ scheduler to correctly distinguish and compare the timestamps of two different packets. ${ }^{3}$ From Theorem 2, we have

$$
m \geq \log _{2}\left\{\frac{(\beta+e) h^{*}+\alpha^{-1}(\beta+e+\Delta)\left\{1-(1-\alpha)^{H^{*}-h^{*}}\right\}}{\left((1-\alpha)^{H^{*}-h^{*}-1}-\alpha h^{*}\right) \Gamma}\right\}+1 .
$$

From (9), we see that, for a fixed network utilization level $\alpha$, larger $\Gamma$ may reduce the number of bits needed for packet timestamp encoding. However, as we increase $\Gamma, h^{*}$ may also be increased. Consequently, the right-hand side of (9) may increase. Hence, the relationship between $m$ and $\Gamma$ is not strictly monotone. Furthermore, a larger $\Gamma$, in general, also yields a smaller maximum allowable network utilization level bound.

Now, let us derive the number of bits needed to encode the timestamp information in a network of $\operatorname{SETF}(0)$ schedulers. Suppose that $C^{*}$ is the maximum link capacity of the network. Then, it is sufficient to have a time granularity of $\iota=1 / C^{*}$ to mark the precise time each bit of data enters the network. ${ }^{4}$ In other words, $\iota=1 / C^{*}$ is the finest time granularity needed to represent packet timestamps. In the remainder of this paper, we will assume that the clock granularity of the edge devices that place timestamps on packets entering the network is at least $\iota$, i.e., the clocks tick (at least) every $\iota$ units of time. Following the same argument as above and from Theorem 3, we have the number of bits, $m$, that is needed to encode the timestamp information in $\operatorname{SETF}(0)$

$$
m \geq \log _{2}\left\{\frac{\alpha^{-1}(\beta+e+\Delta)\left\{1-(1-\alpha)^{H^{*}}\right\}}{\left((1-\alpha)^{H^{*}-1}\right) \iota}\right\}+1 .
$$

\subsection{Numerical Studies}

In this section, we perform numerical studies to illustrate the performance trade-offs and provisioning power of SETF schedulers. In all the studies, we assume that the capacity of all links is $10 \mathrm{~Gb} / \mathrm{s}$ and all packets have the same size, $L=$ 1,000 bytes. Moreover, we assume that there is only one traffic aggregate, i.e., all the routers are GR nodes for the aggregate with parameters $(10 \mathrm{~Gb} / \mathrm{s}, 0)$ (we make such an assumption in all the following numerical examples). We set the network burstiness factor $\beta$ in a similar manner as in [6]: We assume that the token bucket size of each flow is bounded in such a way that $\sigma^{j} \leq \beta_{0} \rho^{j}$, where $\beta_{0}$ (measured in units of time) is a constant for all flows. For a given network utilization level $\alpha$, we then set $\beta=\alpha \beta_{0}$. In all the

3. Here, we assume that no extra clock or other timing device/ mechanism is used to assist an $\operatorname{SETF}(\Gamma)$ scheduler to distinguish the packet timestamps. In other words, an $\operatorname{SETF}(\Gamma)$ scheduler must use the bit strings encoded in the packet header to determine whether the timestamp of one packet is smaller than that of another packet. This can be achieved, for example, by using the lollipop sequence number technique [10]. Note that if we assume that each $\operatorname{SETF}(\Gamma)$ scheduler has a clock that is synchronized with the edge timestamping devices and, thus, can use this clock to identify the time slot the current time corresponds to, then it is sufficient to have $2^{m} \Gamma \geq D^{*}$, i.e., one less bit is needed in this case.

4. Although, theoretically speaking, the finest time granularity $\Gamma=0$, it is obvious that, in practice, $\iota=1 / C^{*}$ is sufficient as no two bits can arrive at any link within $\iota$ units of time. 


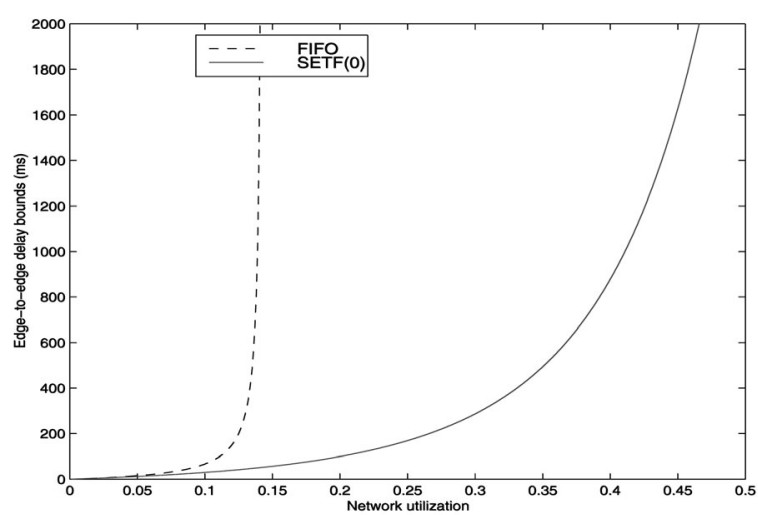

Fig. 4. Performance comparison: SETF(0) versus FIFO.

numerical studies presented in this paper, we choose $\beta_{0}=25 \mathrm{~ms}$.

Fig. 4 compares the worst-case edge-to-edge bounds for a FIFO network and an $\operatorname{SETF}(0)$ network (with $H^{*}=8$ ) as a function of the network utilization level $\alpha$. From Fig. 4, it is clear that, for a given network utilization level, the worstcase edge-to-edge delay bound for an $\operatorname{SETF}(0)$ network is much better than that for a FIFO network. On the other hand, because $\operatorname{SETF}(0)$ needs to encode a fine-grained timestamp information in packet headers, we expect that the cost in terms of the number of bits needed to encode timestamp will be high, as illustrated in Fig. 5. This figure shows the number of bits needed for packet timestamp encoding for two $\operatorname{SETF}(0)$ networks with $H^{*}=8$ and $H^{*}=12$, respectively. The other parameters used in this example are the same as in Fig. 4. In particular, $C^{*}=$ $10 \mathrm{~Gb} / \mathrm{s}$ and, thus, $\iota=1 / \mathrm{C}^{*}=10^{-7} \mathrm{~ms}$. As expected, the number of bits needed for packet timestamp encoding increases as the network utilization level increases; it also increases as the network diameter scales up. From this figure, we also see that, even for a relatively low network utilization level, the number of bits required for packet timestamp encoding is relatively large. For example, with $H^{*}=8,26$ bits are needed for $\alpha=0.1$. Consequently, to achieve a meaningful network utilization level, an SETF(0) network requires a large number of bits for packet timestamp encoding, thus incurring significant control overhead. Below, we conduct numerical studies on $\operatorname{SETF}(\Gamma)$ to

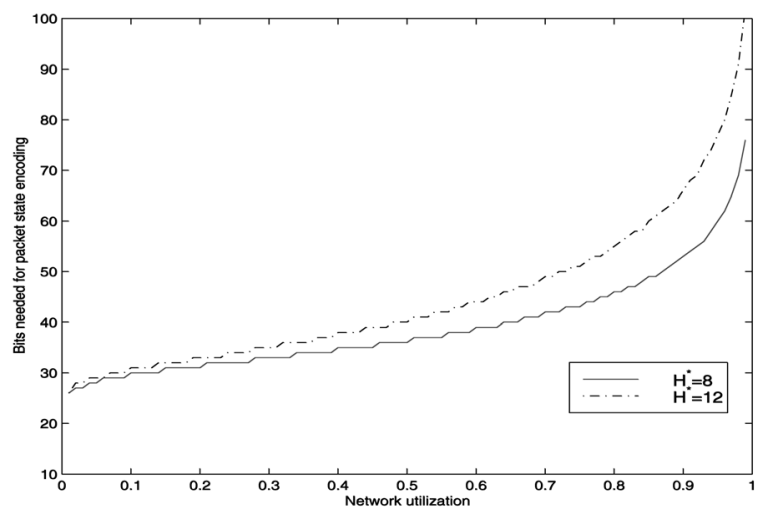

Fig. 5. Number of bits needed for encoding for $\operatorname{SETF}(0)$.

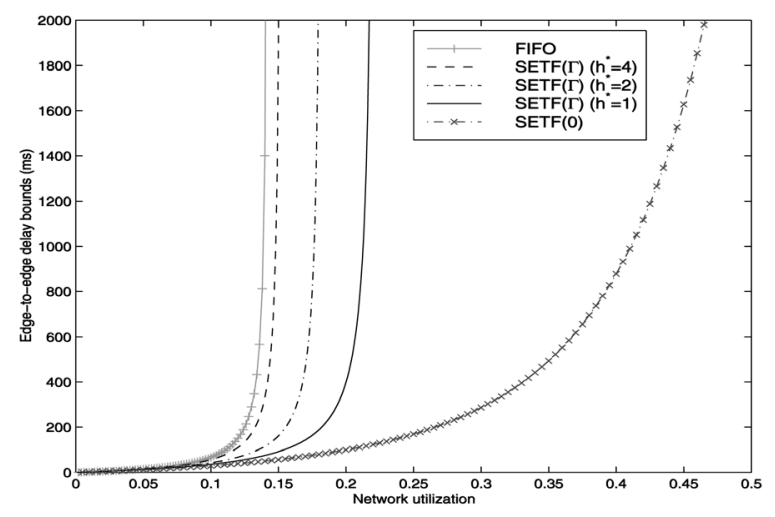

Fig. 6. Performance comparison: SETF versus FIFO.

show how this problem can be potentially addressed by using coarser time granularity for packet timestamp encoding in $\operatorname{SETF}(\Gamma)$. First, we note that, from Theorem 2, (9), and the definition of $h^{*}(5)$, we can see that, given a network with diameter $H^{*}$, we can essentially divide the time granularity $\Gamma$ into $H^{*}$ granularity levels: Each granularity level corresponds to one value of $h^{*}=0,1, \ldots, H^{*}-1$. The finest granularity level corresponds to $h^{*}=0$, and the coarsest granularity level to $h^{*}=H^{*}-1$. For this reason, in the following numerical studies, we will use $h^{*}$ to indicate the time granularity used in an $\operatorname{SETF}(\Gamma)$ network.

Fig. 6 shows the effect of time granularity on the worstcase edge-to-edge delay bound for an $\operatorname{SETF}(\Gamma)$ network with $H^{*}=8$. For comparison, we also include the results for the corresponding FIFO network. From the figure, it is clear that coarser time granularity (i.e., larger $h^{*}$ ) yields poorer worst-case edge-to-edge delay bound. As the time granularity gets coarser (i.e., $h^{*}$ increases), the worst-case edge-toedge delay bound quickly approaches to that of the FIFO network.

Next, we illustrate how the network utilization level of an $\operatorname{SETF}(\Gamma)$ network affects the number of bits needed for packet timestamp encoding. Fig. 7 shows the number of bits needed for packet timestamp encoding as a function of the network utilization level under various time granularities (as indicated by $h^{*}$ ). In this example, the network diameter $H^{*}=8$. From this figure, we see that, for low network utilization levels, using coarser time granularity reduces the

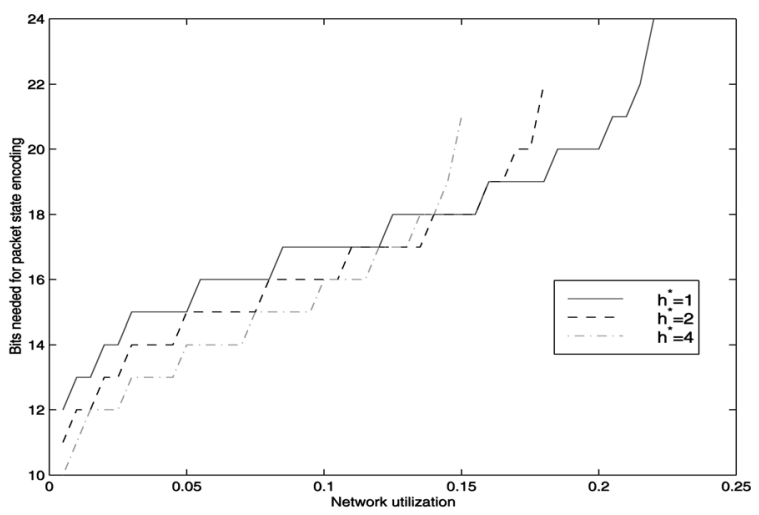

Fig. 7. Number of bits needed for encoding for $\operatorname{SETF}(\Gamma)$. 


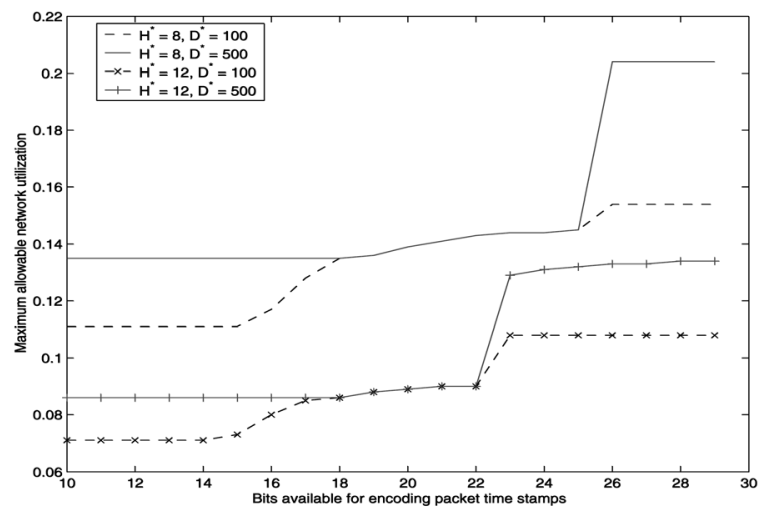

Fig. 8. Number of bits for encoding, network diameter, and maximum allowable network utilization.

number of bits needed for packet timestamp encoding. However, as coarser time granularity also imposes a tight bound on the maximum allowable network utilization, this reduction in the number of bits needed for packet timestamp encoding may not be feasible when the network utilization level is increased (this is why the curve for a given time granularity $\left(h^{*}\right)$ stops at certain network utilization level). To put it another way, to achieve a higher network utilization level, $\operatorname{SETF}(\Gamma)$ schedulers with finer time granularity (thus, smaller $h^{*}$ ) must be used, thus requiring more bits for packet timestamp encoding.

In the last set of numerical studies, we demonstrate how the number of bits available for packet timestamp encoding affects the maximum allowable network utilization so as to support a given target worst-case edge-to-edge delay bound for SETF networks. The results are shown in Fig. 8, where networks with a combination of the network diameters $H^{*}=8$ and $H^{*}=12$ and delay bounds $D_{H^{*}}=100 \mathrm{~ms}$ and $D_{H^{*}}=500 \mathrm{~ms}$ are used. As we can see from the figure, for a given number of bits for packet timestamp encoding, as the network diameter increases, the maximum allowable network utilization decreases. Note also that, when the number of bits for packet timestamp encoding is small (e.g., less than 15 for a network with parameters $H^{*}=8$ and $\left.D_{H^{*}}=100 \mathrm{~ms}\right)$, the packet timestamp does not enhance the performance of a $\operatorname{SETF}\left(\Gamma, h^{*}\right)$ network and the $\operatorname{SETF}\left(\Gamma, h^{*}\right)$ network behaves essentially as a FIFO network with a maximum network utilization level around 0.11. Beyond this threshold, as the number of bits used increases, the maximum allowable network utilization also increases. However, as the figure shows, further increasing the number of bits beyond a certain value (e.g., 26 for a network with parameters $H^{*}=8$ and $D^{*}=100 \mathrm{~ms}$ ) for encoding will not improve the maximum allowable network utilization.

\section{Network of Dynamic Earliest Time First SCHEDULERS}

So far, we have seen that, by including additional control information in the packet header and adding sophistication/complexity at network schedulers, the class of SETF packet scheduling algorithms improve upon the maximum allowable network utilization and worst-case edge-to-edge

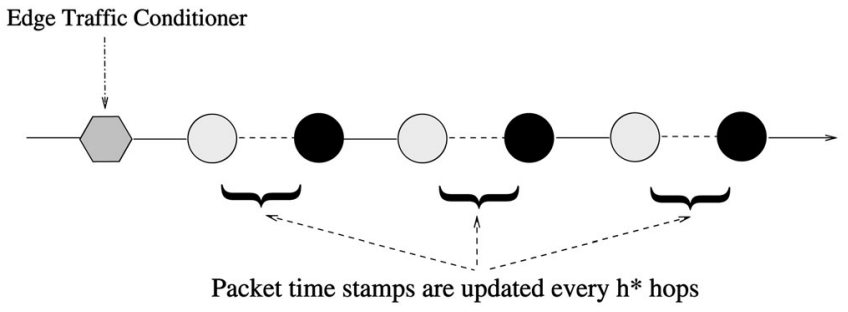

Fig. 9. Updating packet timestamps inside the network core.

delay bounds of the simple FIFO packet scheduling algorithm. This performance improvement comes essentially from the ability of an SETF scheduler to limit the effect of "newer" packets on "older" packets. However, the provisioning power of SETF packet scheduling algorithms is still rather limited. Given the finest time granularity to encode the packet timestamps, although we can achieve arbitrary network utilization in a network of $\operatorname{SETF}(0)$ schedulers, the worst-case edge-to-edge delay bound is inversely proportional to $(1-\alpha)^{H^{*}}$. Hence, the bound grows exponentially, as the network diameter $H^{*}$ increases. In addition, with coarser time granularities, the performance of SETF networks deteriorates further. In this section, we devise another class of aggregate packet scheduling algorithms-the class of DETF algorithmswhich, with further "sophistication/complexity" added at the schedulers, achieve far superior performance.

In the general definition of a DETF packet scheduling algorithm, we use two parameters: the time granularity $\Gamma$ and the (packet) timestamp increment hop count $h^{*}$. Note that, unlike SETF, where $h^{*}$ is determined by $\Gamma$, here, $h^{*}$ is independent of $\Gamma$. Hence, we denote a DETF scheduler by $\operatorname{DETF}\left(\Gamma, h^{*}\right)$. In the following, we will present the definition of $\operatorname{DETF}\left(0, h^{*}\right)$ first, i.e., DETF with the finest time granularity. The general definition of $\operatorname{DETF}\left(\Gamma, h^{*}\right)$ will be given afterward.

As in the case of $\operatorname{SETF}(0)$, the timestamp of a packet in a network of $\operatorname{DETF}\left(0, h^{*}\right)$ schedulers is represented precisely. In particular, it is initialized at the network edge with the time the packet is released into the network. Unlike $\operatorname{SETF}(0)$, however, the timestamp of the packet will be updated every $h^{*}$ hops (see Fig. 9). Formally, suppose packet $p$ traverses a path of $h$ hops. Let $\omega_{0}^{p}$ denote the timestamp of packet $p$ as it is released into the network, i.e., $\omega_{0}^{p}=a_{1}^{p}$. Let $\kappa=\left\lceil\frac{h}{h^{*}}\right\rceil$. For $k=1,2, \ldots, \kappa-1$, the timestamp of packet $p$ is updated after it has traversed the $k h^{*}$ th hop on its path (or as it enters the $\left(k h^{*}+1\right)$ th hop on its path). Let $\omega_{k}^{p}$ denote the packet timestamp of packet $p$ after its $k$ th update. The packet timestamp $\omega_{k}^{p}$ is updated using the following update rule:

$$
\omega_{k}^{p}:=\omega_{k-1}^{p}+d^{*}, \quad k=1, \ldots, \kappa-1,
$$

where the parameter $d^{*}>0$ is referred to as the (packet) timestamp increment. We impose the following condition on $d^{*}$ that relates the packet timestamp $\omega_{k}^{p}$ to the actual time packet $p$ departs the $k h^{*}$ th hop:

$$
\text { for } k=1, \ldots, \kappa-1, \quad f_{k h^{*}}^{p} \leq \omega_{k}^{p} \text {, and } f_{h}^{p} \leq \omega_{\kappa}^{p}:=\omega_{\kappa-1}^{p}+d^{*} .
$$


This condition on $d^{*}$ is referred to as the reality check condition. Intuitively, we can think of the path of packet $p$ being partitioned into $\kappa$ segments of $h^{*}$ hops each (except for the last segment, which may be shorter than $h^{*}$ hops). The reality check condition (12) ensures that the packet timestamp carried by packet $p$ after it has traversed $k$ segments is not smaller than the actual time it takes to traverse those segments. In the next section, we will see that the reality check condition (12) and the packet timestamp update rule (11) are essential in establishing the performance bounds for a network of DETF schedulers.

We now present the definition for the general $\operatorname{DETF}\left(\Gamma, h^{*}\right)$ packet scheduling algorithm with a (coarser) time granularity $\Gamma>0$. As in the case of $\operatorname{SETF}(\Gamma)$, in a network of $\operatorname{DETF}\left(\Gamma, h^{*}\right)$ schedulers, the time is divided into time slots of $\Gamma$ units: $[(n-1) \Gamma, n \Gamma), n=1,2, \ldots$, and all packet timestamps are represented using the time slots. In particular, if packet $p$ is released into the network in the time slot $[(n-1) \Gamma, n \Gamma)$, then $\omega_{0}^{p}=n \Gamma$. We also require that the packet timestamp increment $d^{*}$ be a multiple of $\Gamma$. Hence, the packet timestamp $\omega_{k}^{p}$ is always a multiple of $\Gamma$. In practice, we can encode $\omega_{k}^{p}$ as the corresponding time slot number (as in the case of $\operatorname{SETF}(\Gamma)$ ).

\subsection{Performance Bounds for a Network of DETF Schedulers}

In this section, we establish performance bounds for a network of DETF schedulers. In particular, we will show that, by using dynamic packet timestamps, we can obtain significantly better performance bounds for a network of DETF schedulers than those for a network of SETF schedulers.

Consider a network of $\operatorname{DETF}\left(\Gamma, h^{*}\right)$ schedulers, where $\Gamma \geq 0$ and $1 \leq h^{*} \leq H^{*}$. We first establish an important lemma which bounds the amount of traffic carried by packets at a $\operatorname{DETF}\left(\Gamma, h^{*}\right)$ scheduler whose timestamp values fall within a given time interval. Consider a $\operatorname{DETF}\left(\Gamma, h^{*}\right)$ scheduler $S$. Given a time interval $[\tau, t]$, let $\mathcal{M}$ be the set of packets that traverse $S$ at some time whose timestamp values fall within $[\tau, t]$. Namely, $p \in \mathcal{M}$ if and only if, for some $k=1,2, \ldots, \kappa, S$ is on the $k$ th segment of packet $p^{\prime}$ s path and $\tau \leq \omega_{k-1}^{p} \leq t$. For any $p \in \mathcal{M}$, we say that packet $p$ virtually arrives at $S$ during $[\tau, t]$. Let $\tilde{A}_{S}(\tau, t)$ denote the total amount of traffic virtually arriving at $S$ during $[\tau, t]$, i.e., the total amount of traffic carried by packets in $\mathcal{M}$. Then, we have the following bound on $\tilde{A}_{S}(\tau, t)$.

Lemma 4. Consider an arbitrary GR scheduler $S$ with parameters $\left(r_{S}, e_{S}\right)$ in a network of $\operatorname{DETF}\left(\Gamma, h^{*}\right)$ schedulers. For any time interval $[\tau, t]$, let $\tilde{A}(\tau, t)$ be defined as above. Then,

$$
\tilde{A}(\tau, t) \leq \beta r_{S}+\alpha r_{S}(t-\tau+\Gamma)
$$

Proof. For simplicity, we first prove a bound on $\tilde{A}^{j}(\tau, t)$, the amount of traffic virtually arriving at $S$ during $[\tau, t]$ from a flow $j$. Consider an arbitrary packet $p$ of flow $j$ which virtually arrives at $S$ (on the $k$ th segment) during $[\tau, t]$, i.e., $\tau \leq \omega_{k-1}^{p} \leq t$. From (11), it is easy to see that

$$
\omega_{k-1}^{p}=\omega_{0}^{p}+(k-1) d^{*} .
$$

Because $\tau \leq \omega_{k-1}^{p} \leq t$, we have

$$
\tau-(k-1) d^{*} \leq \omega_{0}^{p} \leq t-(k-1) d^{*} .
$$

Therefore,

$$
\begin{aligned}
\tilde{A}^{j}(\tau, t) & \leq \sigma^{j}+\rho^{j}\left\lceil\frac{t-(k-1) d^{*}-\left(\tau-(k-1) d^{*}\right)}{\Gamma}\right\rceil \Gamma \\
& \leq \sigma^{j}+\rho^{j}(t-\tau+\Gamma) .
\end{aligned}
$$

From (14) and the edge traffic provisioning condition (2), the lemma follows easily.

Note that if $\Gamma=0$, the bound on $\tilde{A}(\tau, t)$ is exactly the same as the edge traffic provisioning condition (2). Intuitively, (13) means that using the (dynamic) packet timestamp with the finest time granularity, the amount of traffic virtually arriving at $S$ during $[\tau, t]$ is bounded in a manner as if the traffic were reshaped at $S$ using (2). In the general case where a coarser time granularity $\Gamma>0$ is used, an extra $\alpha r_{S} \Gamma$ amount of traffic may (virtually) arrive at $S$, as opposed to (2) at the network edge. This is not surprising since, with a coarser time granularity, a scheduler $S$ inside the network core cannot distinguish a packet from those other packets that traverse $S$ and have the same timestamp value.

From Lemma 4, we can derive a recursive relation for $\omega_{k}^{p \prime}$ s using a similar argument as used before. Based on this recursive relation, we can establish performance bounds for a network of $\operatorname{DETF}\left(\Gamma, h^{*}\right)$ schedulers. The general results are somewhat "messy" to state. For brevity, in the following, we present results for three special but representative cases. As we will see later, the first two theorems are sufficient to demonstrate the provisioning power of a network of DETF schedulers. The third theorem is included here for comparison purpose. Their proofs can be found in [13].

Theorem 5 (A Network of GR-DETF(0, 1) Schedulers). Consider a network of $\operatorname{GR}-\operatorname{DETF}(0,1)$ schedulers with a network diameter $H^{*}$. Let $d^{*}=\beta+e+\Delta$, then the reality condition (12) holds. Furthermore, for any $0<\alpha<1$, the worst-case edge-to-edge delay $D^{*}$ is bounded above by $D^{*} \leq H^{*} d^{*}=H^{*}(\beta+e+\Delta)$.

Theorem 6 (A Network of GR-DETF $(\Gamma, 1)$ Schedulers). Consider a network of GR-DETF $(\Gamma, 1)$ schedulers with a network diameter $H^{*}$, where $\Gamma>0$. Let $d^{*}=\lceil(\alpha \Gamma+\beta+e+\Delta) / \Gamma\rceil \Gamma$, then the reality condition (12) holds. Furthermore, for any $0<\alpha<1$, the worst-case edge-to-edge delay $D^{*}$ is bounded above by $D^{*} \leq H^{*} d^{*}+\Gamma$.

Theorem 7 (A Network of GR-DETF $\left(\Gamma, h^{*}\right)$ Schedulers with $\left.d^{*}=\Gamma\right)$. Consider a network of $\operatorname{GR}-\operatorname{DETF}\left(\Gamma, h^{*}\right)$ schedulers with a network diameter $H^{*}$, where $\Gamma>0$ (and $h^{*}>1$ ). We set $d^{*}=\Gamma$, i.e., the packet timestamp is advanced exactly one time slot every time it is updated. Let $\kappa^{*}=\left\lceil H^{*}\right\rceil$. Suppose the network utilization level $\alpha$ and the time granularity $\Gamma$ satisfy the following condition:

$$
0<\frac{h^{*}(\alpha \Gamma+\beta+e+\Delta)}{1-\left(h^{*}-1\right) \alpha} \leq d^{*}=\Gamma .
$$

Then, the worst-case edge-to-edge delay $D^{*}$ is bounded above by $D^{*} \leq\left(\kappa^{*}+1\right) \Gamma$. 
From Theorem 5 and Theorem 6, we see that, with $h^{*}=1$, the worst-case edge-to-edge delay bound is linear in the network diameter $H^{*}$. Furthermore, with the finest time granularity, the worst-case edge-to-edge delay bound is independent of the network utilization level $\alpha$. This is because the per-hop delay is bounded by $d^{*}=\beta+e+\Delta$. With a coarser time granularity $\Gamma>0$, the per-hop delay is bounded by $d^{*}=\lceil(\alpha \Gamma+\beta+e+\Delta) / \Gamma\rceil \Gamma$, where the network utilization level determines the "additional delay" $(\alpha \Gamma)$ that a packet may experience at each hop.

From Theorem 7, we see that, in a network of $\operatorname{DETF}\left(\Gamma, h^{*}\right)$ where $d^{*}=\Gamma$ and $h^{*}>1$, the maximum allowable network utilization is bounded. To see why this is the case, first note that we must have $\alpha<1 /\left(h^{*}-1\right)$; otherwise, the left-hand side of (15) becomes infinity. For a given $\Gamma>0$, (15) imposes the following tighter bound on $\alpha$ :

$$
\alpha<\frac{1-h^{*}(\beta+e+\Delta) \Gamma^{-1}}{2 h^{*}-1}<\frac{1}{2 h^{*}-1}<\frac{1}{h^{*}-1} .
$$

For a given $\alpha$ that satisfies (16), comparing the worst-case edge-to-edge delay bound in Theorem 7 to that of a network of FIFO schedulers with a network diameter $h^{*}$, we see that updating packet timestamps every $h^{*}$ hops effectively reduces a network of diameter $H^{*}$ into a number of smaller networks with diameter $h^{*}$. In particular, setting $d^{*}=\Gamma$ allows us to consider these smaller networks as networks of FIFO schedulers with diameter $h^{*}$. By appropriately taking into account the effect of dynamic timestamps with coarser time granularity (the extra $\alpha \Gamma+\Delta$ factor), Theorem 7 can essentially be obtained from the bound for a network of FIFO schedulers.

\subsection{Packet State Encoding}

In this section, we first consider the problem of packet state encoding for a network of DETF schedulers, namely, the number of bits that is needed to encode the dynamic packet timestamp and possibly other control information for the proper operation of a DETF network.

First, consider a network of $\operatorname{DETF}(0,1)$ schedulers with a network diameter $H^{*}$. As in the case of $\operatorname{SETF}(0)$, we use $\iota$ to denote the finest time granularity necessary to represent the packet timestamps, i.e., $\iota=1 / C^{*}$, where $C^{*}$ is the maximum link capacity of the network. From Theorem 5, we see that the number of bits $m$ that is needed to encode the (dynamic) packet timestamps precisely must satisfy the following condition:

$$
\begin{aligned}
& 2^{m-1} \iota \geq H^{*}(\beta+e+\Delta) \text { or } \\
& m \geq \log _{2} H^{*}+\log _{2}[(\beta+e+\Delta) / \iota]+1 .
\end{aligned}
$$

Now, consider a network of $\operatorname{DETF}(\Gamma, 1)$ with a coarser time granularity $\Gamma>0$. From Theorem 6 , for a given network utilization level $\alpha$, we see that the number of bits $m$ that is needed to encode the (dynamic) packet timestamps must satisfy the following condition:

$$
\begin{aligned}
& 2^{m-1} \Gamma \geq H^{*}\left\lceil\frac{\alpha \Gamma+\beta+e+\Delta}{\Gamma}\right\rceil \Gamma+\Gamma \text { or } \\
& m \geq \log _{2}\left\{H^{*}\left\lceil\frac{\alpha \Gamma+\beta+e+\Delta}{\Gamma}\right\rceil+1\right\}+1 .
\end{aligned}
$$

Hence, for a given network utilization level $\alpha$, coarser time granularity (i.e., larger $\Gamma$ ) in general leads to fewer bits needed to encode the dynamic packet timestamps. However, due to the ceiling operation in (18), at least $\log _{2}\left\{H^{*}+\right.$ $1\}+1$ bits are needed. This effectively places a bound on the range of time granularities that should be used, i.e., $\Gamma \in[0,(\beta+e+\Delta) /(1-\alpha)]$. Any coarser time granularity $\Gamma>(\beta+e+\Delta) /(1-\alpha)$ will not reduce the minimum number of bits, $\log _{2}\left\{H^{*}+1\right\}+1$, needed for packet timestamp encoding.

In the general case where $h^{*}>1$, in order to ensure a $\operatorname{DETF}\left(\Gamma, h^{*}\right)$ scheduler to work properly, not only do we need to encode the packet timestamps, we also need some additional control information to be carried in the packet header of each packet: In order for a scheduler to know whether the packet timestamp of a packet must be updated, we include a hop-count counter as part of the packet state carried in the packet header to record the number of hops a packet has traversed. This hop-count counter is incremented every time a packet traverses a scheduler and it is reset when it reaches $h^{*}$. Thus, the hop-count counter can be encoded using $\log _{2} h^{*}$ number of bits. Therefore, for a network of $\operatorname{DETF}\left(\Gamma, h^{*}\right)$ where $d^{*}$ is set to $\Gamma$, from Theorem 7, the total number of bits needed for packet state encoding is given by

$$
m \geq \log _{2}\left\{\kappa^{*}+1\right\}+1+\log _{2} h^{*},
$$

provided that the network utilization level $\alpha$ and the time granularity $\Gamma$ are chosen in such a manner that (15) holds. Note that, from (19), we have $m \geq \log _{2}\left\{\kappa^{*} h^{*}+h^{*}\right\}+1 \geq$ $\log _{2}\left\{H^{*}+h^{*}\right\}+1$. Therefore, the number of bits needed for encoding the packet states is increased as $h^{*}$ increases. Moreover, via (15), $h^{*}$ also affects the maximum allowable network utilization bound. In particular, from (16), a larger $h^{*}$ leads to a smaller bound on the maximum allowable network utilization. For these reasons, it is sufficient to only consider networks of $\operatorname{DETF}(\Gamma, 1)$ schedulers. ${ }^{5}$

\subsection{Performance Trade-Offs and Provisioning Power of Aggregate Packet Scheduling}

In this section, we use numerical examples to demonstrate the performance trade-offs in the design of DETF networks. By comparing the performance of FIFO, SETF, and DETF networks, we also illustrate the provisioning power of the aggregate packet scheduling algorithms in support of guaranteed delay service. Last, we briefly touch on the issue of complexity/cost in implementing the aggregate packet scheduling algorithms.

The network setting for all the studies is the same as before. Namely, all links have a capacity of $10 \mathrm{~Gb} / \mathrm{s}$, all packets have a size of $L=1,000 B$, and $\beta=\alpha \beta_{0}$, where $\alpha$ is the network utilization level and $\beta_{0}=25 \mathrm{~ms}$. Moreover, as before, only one traffic aggregate is supported by the network for simplicity. The network diameter $H^{*}$ and the network utilization level $\alpha$ will be varied in different studies.

5. In practice, it is possible to implement the hop-count counter using, say, the TTL field in the IP header, thus avoiding the extra $\log _{2} h^{*}$ bits. For example, we have implemented two versions of DETF packet scheduling algorithms in FreeBSD: one using IP-IP tunneling technique, another using MPLS. In both cases, we only need additional bits to encode the packet timestamps. In such situations, a network of $\operatorname{DETF}\left(\Gamma, h^{*}\right)$ schedulers with $d^{*}=\Gamma>0$ and $h^{*}>1$ requires only $\log _{2} \kappa^{*}+1$ additional number of bits. 


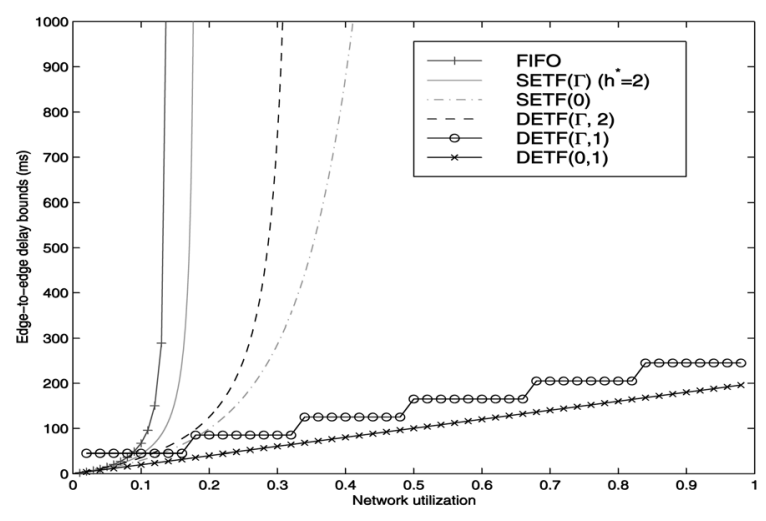

Fig. 10. Edge-to-edge delay bound comparison $\left(H^{*}=8\right)$.

In the first set of numerical examples, we illustrate the relationship between the network utilization level $\alpha$ and the worst-case edge-to-edge delay bound for networks employing various aggregate packet scheduling algorithms. The results are shown in Fig. 10, where $H^{*}=8$ is used for all the networks. For the $\operatorname{SETF}(\Gamma)$ network, we choose $\Gamma=2 \Delta=$ $0.8 \mu$ s (i.e., $h^{*}=2$ ), whereas, in the $\operatorname{DETF}(\Gamma, 2)$ network, the time granularity $\Gamma$ is chosen in such a way that (15) in Theorem 7 holds. For the $\operatorname{DETF}(\Gamma, 1)$ network, we set $\Gamma=5 \mathrm{~ms}$. From the figure, we see that the $\operatorname{DETF}(0,1)$ network has the best worst-case edge-to-edge delay bound. Despite a relatively coarser time granularity, the delay bound for the $\operatorname{DETF}(\Gamma, 1)$ network is fairly close to that of the $\operatorname{DETF}(0,1)$ network. In addition, when the network utilization level is larger than 0.2 , the $\operatorname{DETF}(\Gamma, 1)$ network also has a better delay bound than the rest of the networks. From Theorem 6, it is clear that the worst-case edge-to-edge delay bound for a $\operatorname{DETF}(\Gamma, 1)$ network decreases (and approaches to that of a $\operatorname{DETF}(0,1)$ network), when finer time granularity (smaller $\Gamma$ ) is used. The delay bound of the $\operatorname{DETF}(\Gamma, 2)$ network is worse than that of the $\operatorname{SETF}(0)$ network (with the finest time granularity), but is considerably better than those of the $\operatorname{SETF}(\Gamma)$ and FIFO networks. From this example, we see that the DETF networks, in general, have far better delay performance than those of SETF and FIFO networks.

In the next set of numerical examples, we compare the provisioning power of the various aggregate packet scheduling algorithms. In particular, we consider the following provisioning problem: Given a network employing a certain aggregate packet scheduling algorithm, what is the maximum allowable network utilization level we can attain in order to meet a target worst-case edge-to-edge delay bound? In this study, we allow networks employing different aggregate packet scheduling algorithms to use different number bits for packet state encoding. More specifically, the FIFO network needs no additional bits. The $\operatorname{SETF}(\Gamma)$ network (where $\Gamma$ is chosen such that $h^{*}=1$ ) uses 20 additional bits for timestamp encoding. The number of additional bits used by the $\operatorname{DETF}(\Gamma, 2)$ network is 3 . For the $\operatorname{DETF}(\Gamma, 1)$ networks, we consider two cases: One uses six additional bits, while the other uses 7 bits. All the networks used in these studies have the same diameter $H^{*}=8$. Fig. 11 shows the maximum allowable network utilization level as a function of the target worst-case edgeto-edge delay bound for the various networks. The results clearly demonstrate the performance advantage of the

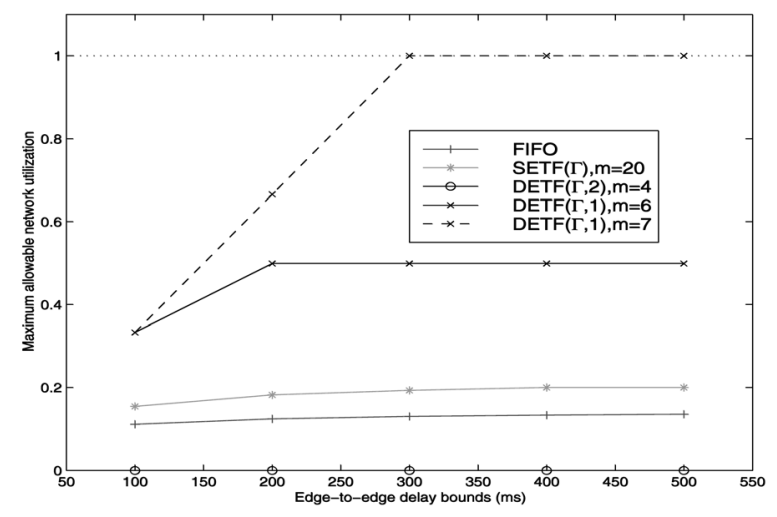

Fig. 11. Provisioning power of $\operatorname{FIFO}, \operatorname{SETF}(\Gamma), \operatorname{DETF}(\Gamma, 1)$, and $\operatorname{DETF}(\Gamma, 2)$ networks $\left(H^{*}=8\right)$.

DETF networks. In particular, with a few number of bits needed for packet state encoding, the $\operatorname{DETF}(\Gamma, 1)$ networks can attain much higher network utilization level, while supporting the same worst-case edge-to-edge delay bound.

In the last set of numerical examples, we focus on the $\operatorname{DETF}(\Gamma, 1)$ networks only. In this study, we investigate the design and performance trade-offs in employing $\operatorname{DETF}(\Gamma, 1)$ networks to support guaranteed delay service. In particular, we consider the following problem: Given a fixed number of bits for packet state encoding, what is the maximum allowable network utilization level that we can attain to support a target worst-case edge-to-edge delay bound? Note that, for a network of diameter $H^{*}$, at least $\log _{2}\left\{H^{*}+\right.$ $1\}+1$ bits are needed for packet state encoding. More bits available will allow us to choose finer time granularity for timestamp encoding, thus yielding a better delay bound as well as a higher maximum network utilization level. In Fig. 12, we show, for a network of diameter $H^{*}=8$, how the number of bits available for packet state encoding affects the maximum network utilization level so as to support a given target worst-case edge-to-edge delay bound. The same results for a network of diameter $H^{*}=12$ are shown in Fig. 13. From these results, we see that, with a relatively few number of bits, a DETF network can achieve fairly decent or good network utilization while meeting the target worst-case edge-to-edge delay bound. In particular, with the target worst-case edge-to-edge delay bounds $200 \mathrm{~ms}$ and $500 \mathrm{~ms}$, we can achieve more than 50 percent (and up to

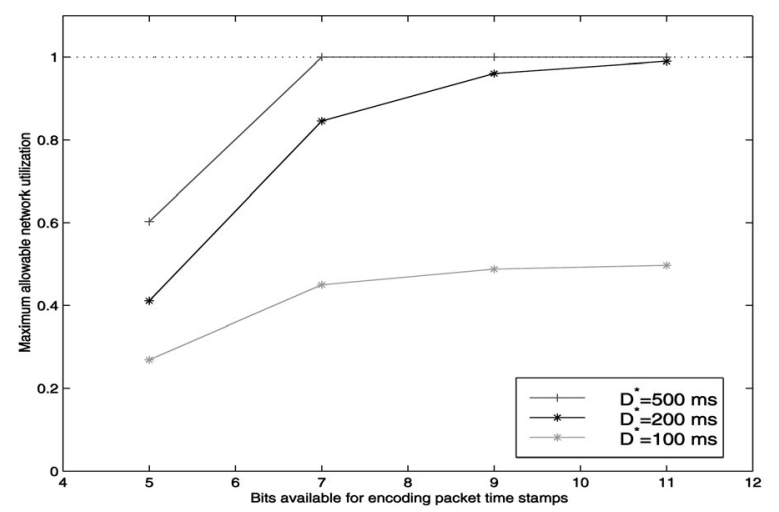

Fig. 12. Design and performance trade-offs for $\operatorname{DETF}(\Gamma, 1)$ networks $\left(H^{*}=8\right)$. 


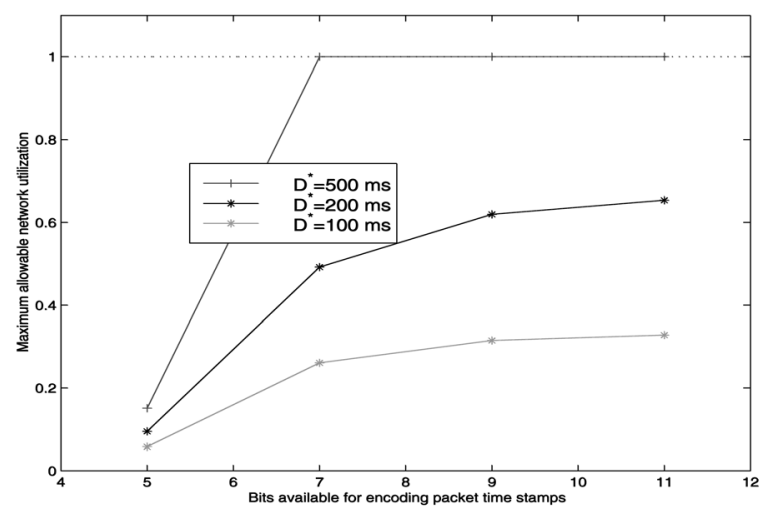

Fig. 13. Design and performance trade-offs for $\operatorname{DETF}(\Gamma, 1)$ networks $\left(H^{*}=12\right)$.

100 percent) network utilization level using only 6 to 7 additional bits. Comparing Fig. 12 and Fig. 13, it is clear that a network with larger diameter requires more bits than a network with smaller diameter to achieve the same maximum allowable network utilization. However, the minimum number of bits required for packet state encoding grows only logarithmically with the network diameter $H^{*}$. Furthermore, today's networks tend to be more "dense," i.e., with relative small $H^{*}$. Hence, with a relatively small number of additional bits (e.g., 8 or 16 bits) for timestamp encoding, we can design $\operatorname{DETF}(\Gamma, 1)$ networks to attain fairly high network utilization while supporting reasonably good edge-to-edge delay bounds.

We conclude this section by briefly touching on the issue of cost/complexity in implementing the aggregate packet scheduling algorithms. Besides the fact that additional bits are needed for packet state encoding, both the SETF and DETF packet scheduling algorithms require comparing packet timestamps and sorting packets accordingly. With the finest time granularity, this sorting operation can be expensive. However, with only a few bits used for packet timestamp encoding, sorting can be avoided by implementing a "calendar queue" (or rotating priority queue [11]) with a number of FIFO queues. This particularly favors the $\operatorname{DETF}(\Gamma, 1)$ packet scheduling algorithms since the number of bits needed for timestamp encoding can be kept small. However, compared to $\operatorname{SETF}, \operatorname{DETF}(\Gamma, 1)$ packet scheduling algorithms require updating packet timestamps at every router and, thus, $d^{*}$ must be configured at each router. Last, in terms of finding additional bits for packet state encoding, we can reuse certain bits in the IP header [12]. This is the case in our prototype implementation using the IP-IP tunneling technique, where we reuse the IP identification field (16 bits) in the encapsulating IP header to encode the packet timestamp.

\section{Conclusions and Future Work}

In this paper, we investigated the fundamental trade-offs in aggregate packet scheduling for support of (worst-case) guaranteed delay service. Based on a novel analytic approach that focuses on network-wide performance issues, we studied the relationships between the worst-case edgeto-edge delay, the maximum allowable network utilization level, and the "sophistication/complexity" of aggregate packet scheduling employed by a network. We designed two new classes of aggregate packet scheduling algor- ithms-the static earliest time first (SETF) and dynamic earliest time first (DETF) algorithms-both of which employ additional timing information carried in the packet header for packet scheduling, but differ in their manipulation of the packet timestamps. Using the SETF and DETF as well as the simple FIFO packet scheduling algorithms, we demonstrated that, with additional control information carried in the packet header and added "sophistication/ complexity" at network schedulers, both the maximum allowable network utilization level and the worst-case edgeto-edge delay bound can be significantly improved. We further investigated the impact of the number of bits available for packet state encoding on the performance trade-offs as well as the provisioning power of these aggregate packet scheduling algorithms. In particular, we showed that, with a relatively small number of bits for packet state encoding, the DETF packet scheduling algorithms can attain fairly good performance bounds. These results illustrate the fundamental trade-offs in the design of aggregate packet scheduling algorithms and shed light on the provisioning power of aggregate packet scheduling in support of guaranteed delay service.

There are a number of research directions we are currently exploring. By taking into account the actual network topology, we are extending the analytic approach presented in this paper to obtain better performance bounds. Such an analysis may also help us identify "hot spots" and "bottleneck" links in a network and, therefore, allow us to possibly make special provisioning for network "hot spots" and "bottleneck" links. Using the insights obtained in this paper, we are also studying stochastic traffic behavior in a network with aggregate packet scheduling. Through this study, we hope to obtain useful provisioning rules for providing predictable Internet QoS services (e.g., along the line of [3]). Extensions to the DETF aggregate packet scheduling for supporting multiple delay classes and rate guarantees are also under investigation.

\section{ACKNOWLEDGMENTS}

The authors would like to thank the anonymous reviewers of the Proceedings of the IEEE International Conference on Network Protocols 2001 and the IEEE Transactions on Parallel and Distributed Systems for many valuable comments. This work was supported in part by US National Science Foundation Grants CAREER Award NCR-9734428, EIA9818338, and ITR ANI-0085824. Any opinions, findings, and conclusions or recommendations expressed in this paper are those of the authors and do not necessarily reflect the views of the US National Science Foundation. An earlier, abridged version of this paper appeared in the Proceedings of the IEEE International Conference on Network Protocols 2001 under the same title.

\section{REFERENCES}

[1] J. Bennett, K. Benson, A. Charny, W. Courtney, and J.-Y. Le Boudec, "Delay Jitter Bounds and Packet Scale Rate Guarantee for Expedited Forwarding," Proc. IEEE INFOCOM, Apr. 2001.

[2] S. Blake, D. Black, M. Carlson, E. Davies, Z. Wang, and W. Weiss, "An Architecture for Differentiated Services," RFC 2475, Dec. 1998.

[3] T. Bonald, A. Proutiere, and J. Roberts, "Statistical Guarantees for Streaming Flows Using Expedited Forwarding," Proc. IEEE INFOCOM, Apr. 2001. 
[4] J.-Y. Le Boudec and P. Thiran, Network Calculus. Springer-Verlag, http:/ / lcawww.epfl.ch, July 2001.

[5] C.-S. Chang, A Filtering Theory for Communication Networks. Springer-Verlag, 1999.

[6] A. Charny and J.-Y. Le Boudec, "Delay Bounds in a Network with Aggregate Scheduling," Proc. Workshop Quality of Future Internet Services, Oct. 2000.

[7] R. Cruz, "A Calculus for Network Delay, Part I: Network Elements in Isolation," IEEE Trans. Information Theory, vol. 37, no. 1, pp. 114-131, Jan. 1991.

[8] V. Jacobson, K. Nichols, and K. Poduri, "An Expedited Forwarding PHB," RFC 2598, June 1999.

[9] V. Jacobson, K. Nichols, and K. Poduri, "The 'Virtual Wire' PerDomain Behavior," Internet Draft, July 2000.

[10] S. Keshav, An Engineering Approach to Computer Networking. Addison-Wesley, 1997.

[11] J. Liebeherr and D.E. Wrege, "A Versatile Packet Multiplexer for Quality-of-Service Networks," Proc. Fourth Int'l Symp. High Performance Distributed Computing (HPDC-4), pp. 148-155, Aug. 1995.

[12] I. Stoica and H. Zhang, "Providing Guaranteed Services without Per Flow Management," Proc. ACM SIGCOMM, Sept. 1999.

[13] Z.-L. Zhang, Z. Duan, and Y.T. Hou, "Fundamental Trade-Offs in Aggregate Packet Scheduling," technical report, Computer Science Dept., Univ. of Minnesota, http://www.cs.umn.edu/ $\sim$ zhzhang/papers.html, Feb. 2001.

Zhenhai Duan (S'97-M'03) received the BS degree from Shandong University, China, in 1994, the MS degree from Beijing University, China, in 1997, and the PhD degree from the University of Minnesota, in 2003, all in computer science. He is currently an assistant professor in the Computer Science Department at Florida State University. His research interests include computer networks and multimedia communications, especially scalable network resource control and management in the Internet, Internet routing protocols, service architectures, and networking security. Dr. Duan is a corecipient of the 2002 IEEE International Conference on Network Protocols Best (ICNP) Paper Award. He is a member of the IEEE and ACM.

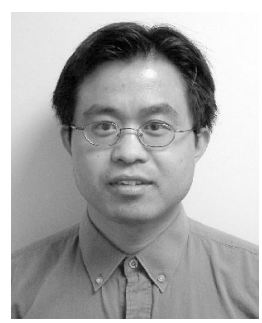

Zhi-Li Zhang (M'97) received the BS degree in computer science from Nanjing University, China, in 1986 and the MS and PhD degrees in computer science from the University of Massachusetts in 1992 and 1997. In 1997, he joined the Computer Science and Engineering faculty at the University of Minnesota, where he is currently an associate professor. From 1987 to 1990, he conducted research in the Computer Science Department at Århus University, Denmark, under a fellowship from the Chinese National Committee for Education. He has held visiting positions at Sprint Advanced Technology Labs, IBM T.J. Watson Research Center, Fujitsu Labs of America, Microsoft Research China, and INRIA, Sophia-Antipolis, France. His research interests include computer communication and networks, especially QoS, routing and security issues in the Internet, multimedia and real-time systems, and modeling and performance evaluation of computer and communication systems. Dr. Zhang currently serves on the editorial board of the IEEE/ ACM Transactions on Networking and Computer Network, an International Journal. He is technical program cochair of IEEE INFOCOM 2006 and the IEEE/IFIP IWQoS '04 as well as the SPIE ITCOM 2002 Conference on Scalability and Traffic Control in IP Networks, served on the executive committee for IEEE Infocom 2001 and Infocom 2003, and on the technical program committees of various conferences and

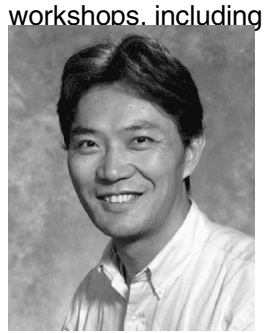

Infocom, IEEE ICNP, ACM SIGCOMM, ACM SIGMETRICS and ACM SIGMM. He received the US National Science Foundation CAREER Award in 1997. He was also awarded the prestigious McKnight Land-Grant Professorship at the University of Minnesota and the Miller Visiting Professorship at the Miller Institute for Basic Sciences, University of California, Berkeley. Dr. Zhang is corecipient of an ACM SIGMETRICS Best Paper Award and an IEEE International Conference on Network Protocols (ICNP) Best Paper Award. He is a member of IEEE, ACM, and INFORMS Telecommunication Section.

Yiwei Thomas Hou (S'91-M'98-SM'04) received the BE degree from the City College of New York in 1991, the MS degree from Columbia University in 1993, and the PhD degree from Polytechnic University, Brooklyn, New York, in 1998, all in electrical engineering. From 1997 to 2002, Dr. Hou was a principal research scientist and project leader at Fujitsu Laboratories of America, IP Networking Research Department, Sunnyvale, California (Silicon Valley). Since Fall 2002, he has been an assistant professor at Virginia Tech, the Bradley Department of Electrical and Computer Engineering, Blacksburg, Virginia. His research interests are in the algorithmic design and optimization for network systems. His current research focuses on wireless ad hoc networks, sensor networks, and video over ad hoc networks. In recent years, he has worked on scalable architectures, protocols, and implementations for differentiated services Internet; service overlay networking; multimedia streaming over the Internet; and network bandwidth allocation policies and distributed flow control algorithms. He has published more than 100 journal and conference papers in the above areas and is a corecipient of the 2002 IEEE International Conference on Network Protocols (ICNP) Best Paper Award and the 2001 IEEE Transactions on Circuits and Systems for Video Technology (CSVT) Best Paper Award. $\mathrm{He}$ is a member of the ACM and a senior member of the IEEE.

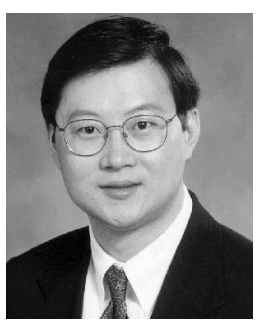

$\triangleright$ For more information on this or any other computing topic, please visit our Digital Library at www.computer.org/publications/dlib. 